\title{
SMALL SCALE RHEOLOGICAL OBSERVATION OF HIGH CONSISTENCY NANOFIBRILLAR MATERIAL BASED FURNISHES
}

\author{
Dimić-Mišić Katarina* \\ Aalto University, Helsinki, Finland \\ Dr Yogesh Sanavane \\ Aalto University, Helsinki, Finland \\ Dr Jouni Paltakari \\ Aalto University, Helsinki, Finland \\ Dr Thaddeus Maloney \\ Aalto University, Helsinki, Finland
}

Nanotechnology allows development of new enabling technologies with broad commercial potential. Cellulose has also the potential to be the source for renewable materials, which can be made multifunctional and self-assembling at the same time displace many non-renewable materials including metal and ceramics. The potential application area includes high quality paper products, paper coatings, high-performance and sustainablecomposite. Nanofibrillated cellulose (NFC) and microfibrillated cellulose (MFC) can be obtained by the mechanical breakdown of natural fibers. These materials have the potential to be produced at low cost in a paper mill and may give many novel properties to paper, paper coatings, paints, or other products. However, suspensions of these materials have a complex rheology even at low solids. To be able to coat, pump, or mix NFC at moderate solids, it is critical to understand the rheology of these suspensions and how they flow in process equipment. Only a limited number of reports have been published in the literature on NFC rheology at moderate solids. NFC was found to be a highly shear thinning material with a power law index of around 0.1. Oscillatory tests gave consistent results for the parallel-disk and other geometries. The complex viscosity, the storage and loss moduli, and the yield stress were determined for the range of solids. Basic rheological and dewatering properties of nanocellulose based high consistency furnishes were evaluated. Two different grades of nanocellulose, microfibrillated cellulose (MFC) and nanofibrillated cellulose (NFC) with different swelling properties were used. Both types of nanocellulose have a common challenge, namely strong interactions between the particles resulting in aggregation of individual particles. These inter-particle interactions together with the physical entanglements cause high viscosities of nanocellulose suspensions and furnishes, which together with high water bonding property affects processability.

Key words: Nanocellulose, Rheology, MFC, NFC, Nanocellulose furnishes

\section{INTRODUCTION}

The properties of wood result from a unique interplay between nanoscale domains of cellulose, hemicellulose, and lignin [06, 14]. In addition to cost, improved environmental friendliness has been a motivation for the use of cellulosecnanosized filler elements in place of other filler materials for the engineering of composite structures [12]. There is huge diversity in raw materials used for productions of nanofibrillar materials and in this work this term will be used for both microfibrillated cellulose (MFC) and nanofibrillatedcellullulose (NFC) [12]. Microfibrillated cellulose (MFC) can be prepared from chemical pulps by mechanical shearing while preserving the chemical composition as well as the high aspect ratio of the cellulose fibrils [25, 35]. An efficient chemical method of producing NFC while preserving high aspect ratio of fibrils is to selectively oxidize primary hydroxyl groups into carboxylate groups via 2, 2, 6, 6-tetramethylpiperdine-1-oxyl 
radical (TEMPO-oxidized) [16, 32, 33]. The hydroxyl groups efficiently prevent the formation of strong inter-fibrillar hydrogen bond-network via electrostatic repulsion $[10,25]$. In respect to production process resulting fibrils have a high aspect ratio and diameter varying between 5 and $100 \mathrm{~nm}$. MFC forms a highly entangled network consisting ofnano scale size elements with a gellike behavior for water dispersions at $1 \%$ or lower concentrations of MFC [01, 15]. In general, nanofibers are elementary assemblies of distinct polymeric units (based on glycopyranose in the central of nanofibrills) that have diameters on the order of tens of nanometers and constitute a fiber/strand network [34].

Since the nanofibrillar material has a fibers carry a negative charge, have a high relative surface area and high surface charge, charge density plays an important role for the interactions between surfaces [08, 20, 33]. Surface charge density of MFC plays an important role for the interactions in paper making furnish, modeling its network strength $[07,20]$ and this effects have been in investigated with microscopy [19, 36]. MFC fibers at certain consistencies form networks and have high shear thinning behavior $[21,28,30]$. Decrease in flock size with increase repulsion between fibers can be explained as more swollen fibers, which have higher bending and water bounded on surface, have lower frictional forces on their wall, enabling fibers to slide past each other in process conditions [36]. This phenomena has been investigated before and seenas decrease in flock size and lower storage modulus (G') $[07,10,11]$. Rheology of paper making furnishes is very important for their processing; pumping, mixing, thickening etc. as observed by many authors [03, 09, 11]. However, amount of nanocellulose and its surface modifications have to be modified in order to balance between positive and negative effects of processability [11, 13].

The rheolocigal complexity of high consistency nanofibrillar material based furnishes was found to be due to the gel-like nature of the nanocellulose conatining material, even so that furnishes are mostly composed of pigment particles amd pulp fibers. This finding suggests of the filler component from a del-like nature of nanocellulose containing material [04, 07, 25]. Further challenges can be due to the moisture absorption by most cellulosic materials, and their tendency to swell when wetted [05].
A key issue that prevents the immediate use of MFC and NFC in a high consistency composites is the rheology of MFC suspensions. Even at a solid level of less than $2 \%$, the behavior is nonNewtonian with difficulty frequently found with a water removal through applications. Therefore, understanding the rheology of these suspensions and furnishes can be of help when tryiung to utilize it more efficiently.

\section{MATERIALS AND METHODS}

\section{Materials}

The charge of the pigments and MFC/ NFCs was determined from the filtrate by a Zetasizernano series (Malvern Instruments Ltd.). The average particle size of the pigments, MFC and NFC was measured with Dynamic Light Scattering (DLS), Malvern (Malvern Instruments Ltd.). Prior to measuring the zeta potential $\left(\Psi_{z}\right)$ and average particle size the samples were diluted with deionized water to a solid content of $0.01 \%$.

The filler added to the furnish was a commercial precipitated calcium carbonate with scalenohedral morphology (SPCC), which came as an undispersed slurry with a solid content of $35.5 \%$ (OmyaOy). The ensemble average particle size of the SPCC was $4.27 \mu_{m}$. The zeta potential was $9.8 \mathrm{mV}$ at $\mathrm{pH} 9$.

Previously dried softwood pine pulp was used as source for cellulose fibers used in this research. Pulp of $360 \mathrm{gr}$ was soaked in $5 \mathrm{I}$ od de-ionized water over night and then beaten to a consistency of 1, 45\% in a Valley Beater (ISO 5264-1) to the Shopper-Riegler (SR) number (24) (ISO 5267-1), zeta potential $-19 \mathrm{mV}$.

Two types of MFC were used for two different furnish grades, Masuko and TEMPO. Masuko MFC Vane viscosity for $10 \mathrm{rpm}$ and $1,5 \%$ consistency was 23176 (mPas), Brookfiledvane viscosity at $0,5 \mathrm{rpm}$ was 489722 (Pas). NFC TEMPO oxidized grade material at consistency of $2,3 \%$ was used, Vane viscosity for $10 \mathrm{rpm}$ at $1,5 \%$ consistency was 48 657(mPas). From initial water suspension of microfibrillated cellulose (MFC) of $3 \%$ and nanofibrillated cellulose (NFC) of $2.3 \%$, other MFC and NFC concentrations ( $2 \%$ and $4 \%$, respectively) were obtainedwith dilution with deionized water and centrifugation in order to increase solid content.

Furnishes were made for three consistencies (4\%, $8 \%$ and $12 \%$, respectively) with solid content with $70 \%$ filler and $30 \%$ pulp. Two different 
recipes were used for each furnish consistency regarding pulp, one with $20 \%$ nanocellulose (and $10 \%$ of cellulose fibers ) and another without cellulose fibers, or $30 \%$ MFC/NFC.WRV for NFC was three time higher than for MFC 9, methods explained elsewhere [07].

\section{Methods}

The rheological measurements were performed at $23^{\circ} \mathrm{C}$ by means of and Anton Paar 300 Rheometer using plate-plate geometry.

Oscillatory measurements were used to characterize the microstructure of the materials. These measurements are usually carried at sufficient small strain amplitude so that the structure is not modified, in linear viscoelastic region (LVE). Viscoelastic behavior was tested onAntonPaar 300 Rheometer using plate-plate geometry, Figure 1. Dynamic moduli, Storage modulus ( $\left.G^{\prime}\right)$ and Loss modulus ( $\left.G^{\prime \prime}\right)$ were measured as a function of frequency $(\omega=0,1-1000 \mathrm{~Hz})$ using oscillatory tests. To perform the frequency sweep test, the linear viscoelastic range of the sample (LVE) was obtained from amplitude sweep using constant angular frequency $\omega=10 \mathrm{~Hz}$ ) with strain amplitude between $(0,01-500 \%)$ [20]. Parallel plate geometry was selected with upper plate diameter $25 \mathrm{~mm}$ where both upper and bottom geometries were roughened (P-PTD 200) in order to prevent slipping of sample [15]. The gap between upper and bottom plate was $1 \mathrm{~mm}$ for suspensions and $1.5 \mathrm{~mm}$ for furnishes. Dynamic viscosity as a function of shear rate was measured at a steady state flow by increasing the shear rate from 0.01 to $1000 \mathrm{~s}-1$. Note that the present geometry is very prone for shear inhomogeneities such as shear banding due to the inherent shear gradient in the radial direction $[27,29]$. This is especially true for shear thinning complex fluids such as nanocellulose. Furthermore, is well documented in the literature, that vacuum filtration induces a gradient in the solid content in the filter normal direction.

Both suspensions and furnishes were measured with a strain sweep, frequency sweep, and steady shear flow. In the strain sweep the strain was from 0.1 to $100 \%$ at an angular frequency $1 \mathrm{~Hz}$. Frequency sweep was done after determining linear viscoelastic region with amplitude sweep, varying angular frequency from 100 to $0.01 \mathrm{~Hz}$.

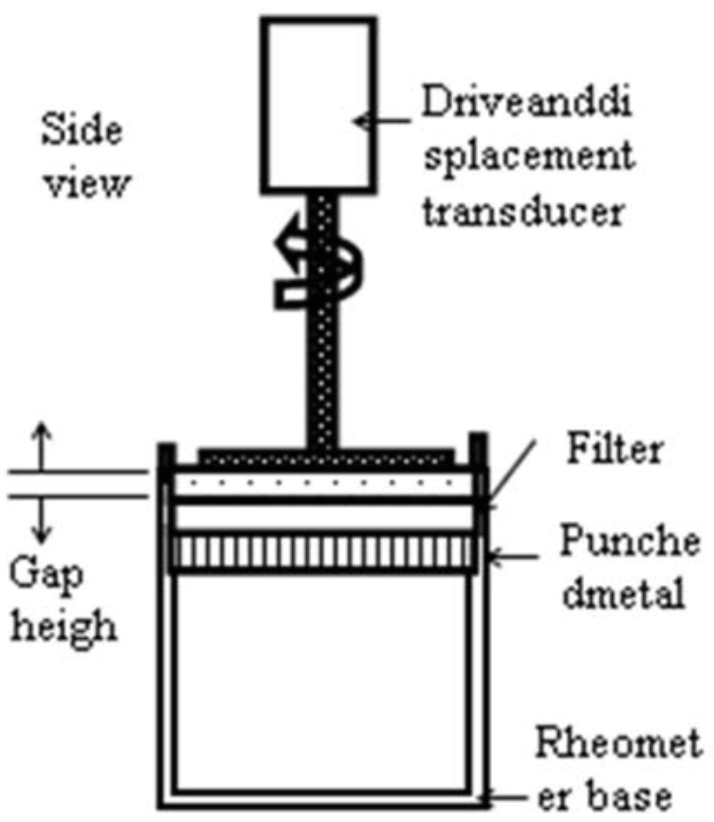

Figure 1: Anton Paar 300 Rheometer

\section{RESULTS AND DISCUSSION}

\section{Suspensions}

Data from strain sweep measurements for suspensions are plotted in Figure 2. Plots presented in Figures 2 a) and b) reports the elastic ( $\left.G^{\prime}\right)$ and viscous ( $\left.G^{\prime \prime}\right)$ moduli and elastic stress as a function of the strain at $1 \mathrm{~Hz}$ for the $2 \%$ and $4 \%$ suspensions, MFC and NFC, respectively. It has been concluded from previous research that swelling is product of increased surface charge of fibers, as zeta potential of fibers affects their swelling properties [10, 07]. Both MFC and NFC suspensions exhibited linear viscoelastic response at low strain. Higher repulsion forces between more swollen NFC suspensions shows as lower elastic modulus $G^{\prime}$ than of MFC suspensions, Figure 2 a). The $G^{\prime}$ is constant up to a critical strain above which the elastic modulus decreases with strain and viscoelastic behavior becomes nonlinear. The $G^{\prime \prime}$ is considerably smaller than the $G^{\prime}$, typical for nanocellulose based gel-likesuspensions.Linear viscoelastic region is longer for NFC suspensions, with critical strain reaching a higher strain values with increase in consistency, due to formation of stronger networks observed from other research papers $[15,07,30]$. Figure 2 b) shows the stress sweep for the storage modulus of different solid contents using the parallel-disk geometry. For strains values lower than $0.01 \%$, both the storage ( $\left.G^{\prime}\right)$ and loss moduli ( $\left.G^{\prime \prime}\right)$ are constant; this result indicates that the materials are in the linear 
viscoelastic regime. The storage modulus $\left(G^{\prime}\right)$ is much higher in magnitude than the loss modulus in these tests, which indicates a solid-like behavior of the material $[02,15,13]$.
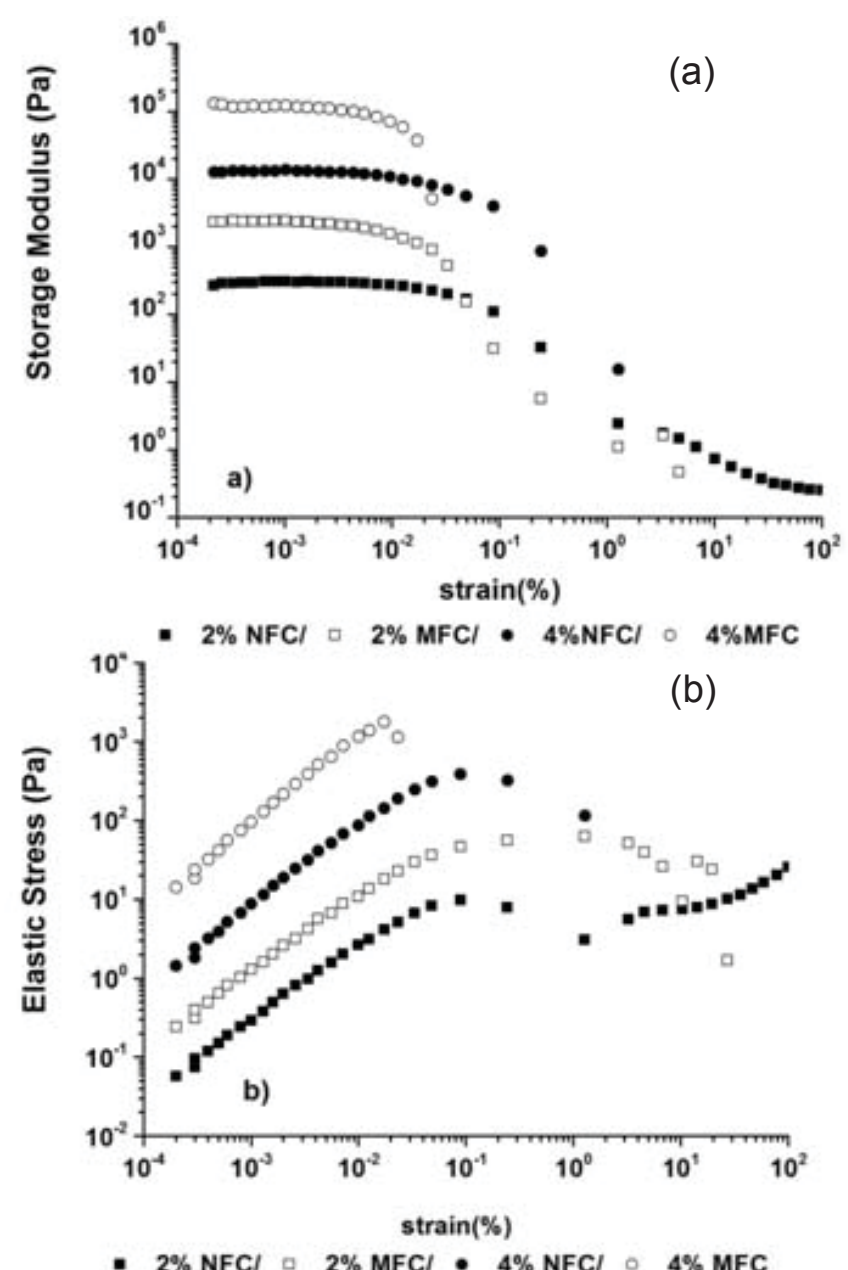

Figure 2: Amplitude at $1 \mathrm{~Hz}$ at strain sweep (0,1-100\%) for MFC and NFC suspentions:

a) Storage and loss moduli for different solids for a range of strains of $1 \mathrm{~Hz}, b)$ Elastic stress as a function of strain for various solids

The steady shear viscosities of NFC suspensions at different solids as a function of shear rate with the parallel-disk geometries are given in Figure 3. The suspensions for all solids show a decrease in viscosity with an increase in shear rate, which is the characteristic ofpronounced shear thinning behavior [30]. As expected, the viscosities increase with increase in solids and follow a shear thinning curve at theconsistent pattern [07]. The slope of the lines are nearly parallel indicating that the power-law exponent are similar for different solidsas observed before [13]. The Cox-Merz rule states that complex viscosity $\left(n^{*}\right)$ versus the angular frequency obtained from small- amplitude oscillatory shear flow should be similar to the steady shear viscosity $(\eta)$ against the shear rate $[23,30]$. Although there is no fundamental explanation for such a relationship, it is widely accepted and used for isotropic polymeric solutions and polymer melts. Figure 3 depicts that the Cox-Merz rule does not hold for MFC suspensions for 2 and $4 \%$ solid, and that they don't have polymer-like behavior, rather being gel-like materials. The complex viscosity is larger in magnitude than the steady shear viscosity data by a large amount. The slopes of the lines are similar for the different solid levels. Other investigations have shown that the complex viscosity is two orders of magnitude larger than the steady shear viscosity. Here, the complex viscosity at certain angular frequencies is over 100 times larger than the steady shear viscosity at the equivalent shear rate-frequency, as observed before for other similar materials [31].

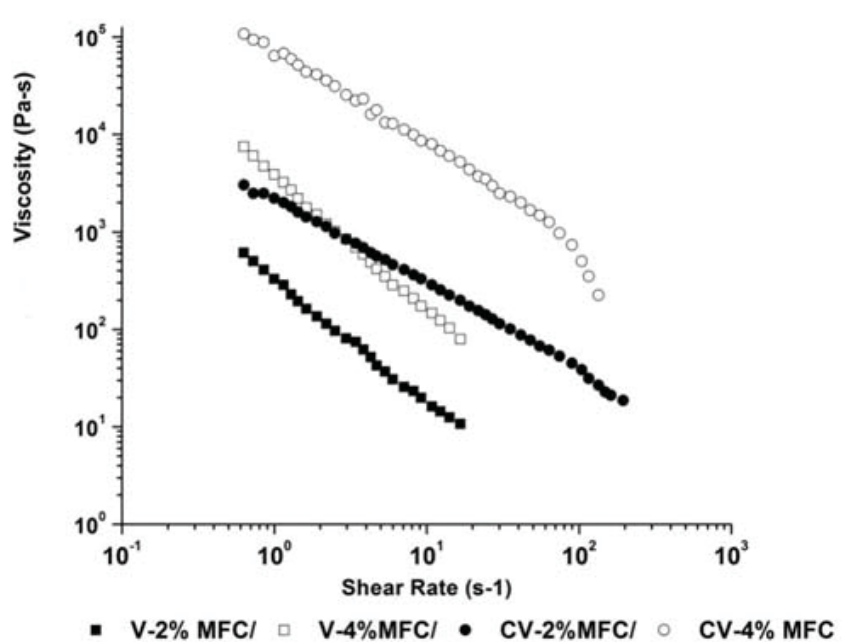

Figure 3: Complex viscosity (CV) and steady viscosity (V) for $2 \%$ and $4 \%$ solids. Each line is an average of three trials

Figure 4 shows the shear stress versus shear rate for MFC and NFC suspensions. All profiles are similar to those presented at Figure 3., with curves showing a minimum with its value at given shear rate.

\section{Furnishes}

Data from frequency sweep measurments form $8 \%$ solids of MFC and NFc furnishes are presented at Figure 5, as representative measurment, similar data are observed for $4 \%$ and $12 \%$ solids. As it can be observed form data ploted for all frequency ranges, $G^{\prime}$ has higher values in comparison to $G$ " which indicates that elastic behaviour dominates the viscous one. 


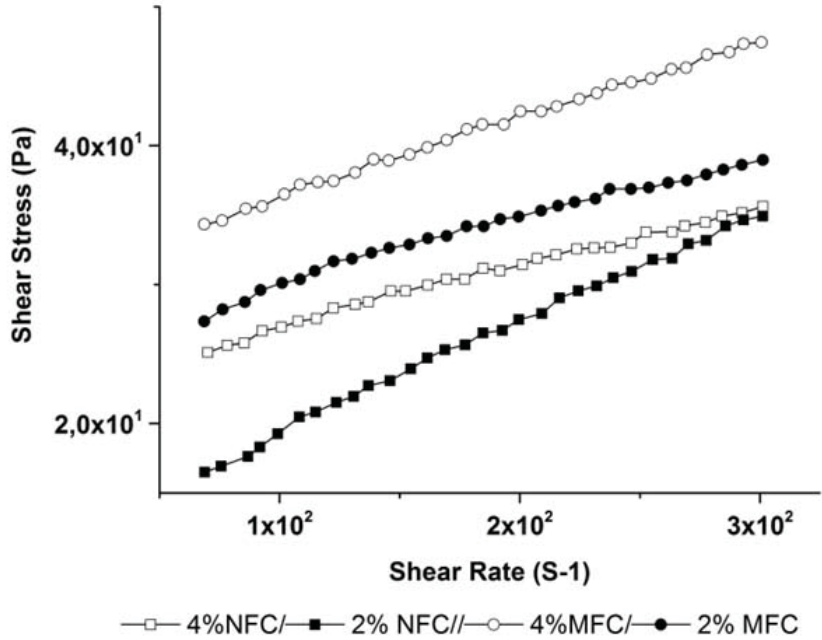

Figure 4: Steady shear stress as a function of shear ratefor $2 \%$ and $4 \%$ solids of MFC and NFC

Typical for gels, intermolecular interaction forces are forming threedimensional networks and thus they are showing $G^{\prime}>G^{\prime}$ ' in the whole frequencyranges with a slight increase in the slope at higher frequencies [17, 18, 37]. Furnishes show high stabilities at low frequencies possibly due to high intermolacular interactions between strong nanocellulose networking within furnish [20]. Elastic moduli for MFC furnishes are sligtly higher than for NFC furnishes, increasing with the presence of cellulose fibers, similar behaviour as seen for suspentions, in Figure 2.

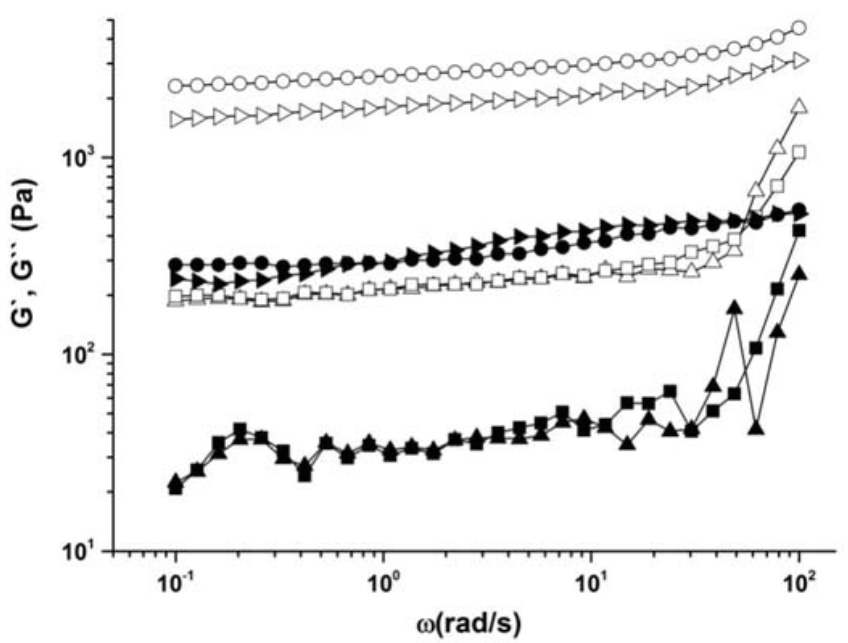

$-8 \% 20 \% \mathrm{NFC} /-8 \% 20 \% \mathrm{NFC} /--8 \% 30 \% \mathrm{NFC} /-8 \% 30 \% \mathrm{NFC}$ $\longrightarrow-8 \% 20 \% \mathrm{MFC} /-8 \% 20 \% \mathrm{MFC} / \longrightarrow 8 \% 30 \% \mathrm{MFC} / \longrightarrow 8 \% 30 \% \mathrm{MFC}$

Figure 5: Elastic (G') and Loss moduli (G') for frequency sweep at $\omega=0,1-100(\mathrm{rad} / \mathrm{s})$ versus for $5 \%$ MFC and NFC furnishes

Figure 6 show profiles of complex viscosity and steady shear viscosity for MFC and NFC furnishes at $12 \%$ solids. Complex viscosity should characterize the resistance to deformation for small deformations. The complex viscosity plotted in Figure 6.a) is obtained from frequency sweep tests and helps to analyze the rheological properties of the suspension. The complex viscosity presented at Figure 6 a) is two orders of magnitude largerthan the steady shear viscosity data presented at Figure $6 \mathrm{~b}$ ). The slopes of the lines are similar for those obtained for suspensions, nicely supporting results from Figure 3. s. It is obvious that for bothnanofibrillar material based suspensions and furnishes CoxMerz rule is not applicable, as stated before [31].
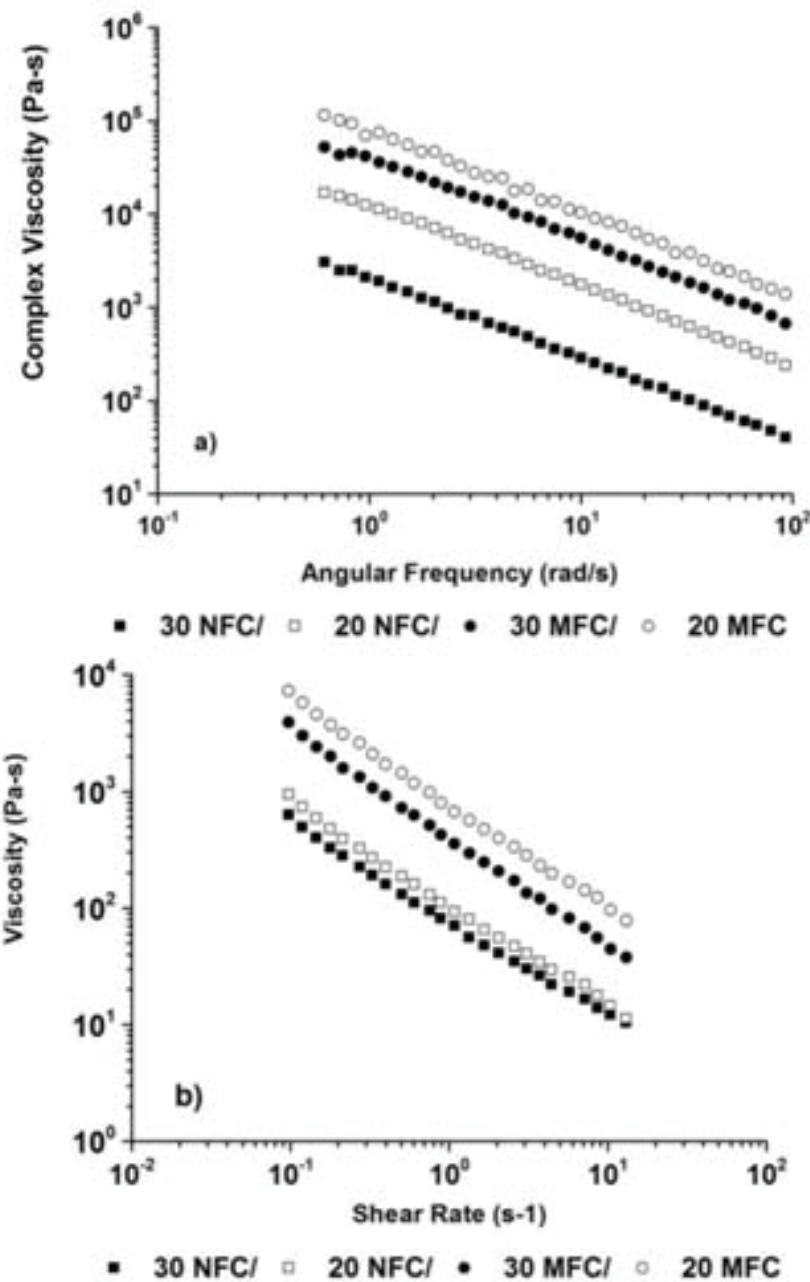

Figure 6: Profiles of viscosities for $12 \%$ furnishes a) complex viscosity profile b) dynamic viscosity profile

\section{CONCLUSIONS}

The rheology of nanofibrillated cellulose is complex with strong shear thinning behavior, significant viscoelastic character and a large yield stress. The complex viscosity at certain angular frequencies is 100 times the steady shear viscosity at equivalent shear rates.

Rheological behavior of nanocellulose is function of its swelling degree.The comparison of both 
dewatering and rheological results indicated that the nanocellulose type, i.e swelling property, had a significant effect on the behavior of furnishes. Rheological behaviorofhigh consistency nanocellulose based furnishes is to a great extent governed by rheological behavior of nanocelluloseapplied in formulation even though furnishes were mostly composed of pigment particles $(70 \%)$ and pulp fibers. This findgs are consistent with hypothesis that swelling influence rheological behaviour of nanocellulose based suspensions and furnishes dominating rheological and dewatering properties. Filler presence, even at very high mass ratio, does not show anyinfluence on the rheological behavior of furnish, filler being trapped within the gel-like furnish matrix. The storage and loss moduli from frequency sweep test showed a gel-like structure for investigated solids of suspensions and furnishes, and dependence of type nanocellulose, i.e. swelling degree. Flow behaviour of high consistency furnishes is the most important property in turbulent conditions in head-box and pipe flow during transportation. Utilizing diffwerent swelling properties of MFC and NFC would make possible to finally predictcomplex rheological behaviour of higly viscous high consistency MFC and NFC furnishes.

\section{REFERENCES}

1) Agoda-Tandjawa, G., Durand, S., Gaillard, C., Garnier, C., \& Doublier, J. -. (2012). Rheological behaviour and microstructure of microfibrillated cellulose suspensions/low-methoxyl pectin mixed systems. effect of calcium ions. Carbohydrate Polymers, 87(2), 1045-1057.

2) Ahola, S., Myllytie, P., Österberg, M., Teerinen, T., \& Laine, J. (2008). Effect of polymer adsorption on cellulose nanofibril water binding capacity and aggregation. BioResources, 3(4), 1315-1328.

3) Ayol, A., Dentel, S. K., \& Filibeli, A. (2010). Rheological characterization of sludges during belt filtration dewatering using an immobilization cell. Journal of Environmental Engineering, 136(9), 992-999.

4) Barnes, H., \& Carnali, J. (1990). The vane-incup as a novel rheometer geometry for shear thinning and thixotropic materials. Journal of Rheology, 34, 841.

5) Bledzki, A., \& Gassan, J. (1999). Composites reinforced with cellulose based fibres. Progress in Polymer Science, 24(2), 221-274.
6) De Morais Teixeira, E., Corrêa, A. C., Manzoli, A., de Lima Leite, F., de Oliveira, C. R., \& Mattoso, L. H. C. (2010). Cellulose nanofibers from white and naturally colored cotton fibers. Cellulose, 17(3), 595-606.

7) Dimić-Mišić, K., \& Paltakari, J. (2012). Fibrillar material as a cobinder in coating colors formulations. Journal of Applied Engineering Science (Istraživanja i projektovanja za privredu), 10(4), 209-220

8) Henriksson, M., Henriksson, G., Berglund, L., \& Lindström, T. (2007). An environmentally friendly method for enzyme-assisted preparation of microfibrillated cellulose (MFC) nanofibers. European Polymer Journal, 43(8), 3434-3441.

9) Heymann, L., Peukert, S., \& Aksel, N. (2002). Investigation of the solid-liquid transition of highly concentrated suspensions in oscillatory amplitude sweeps. Journal of Rheology, 46(1), 93-112.

10) Horvath, A. E., \& Lindström, T. (2007). The influence of colloidal interactions on fiber network strength. Journal of Colloid and Interface Science, 309(2), 511-517.

11) Hubbe, M. A., \& Heitmann, J. A. (2007). Review of factors affecting the release of water from cellulosic fibers during paper manufacture. BioResources, 2(3), 500-533.

12) Hubbe, M. A., Rojas, O. J., Lucia, L. A., \& Sain, M. (2008). Cellulosic nanocomposites: A review. BioResources, 3(3), 929-980.

13) Hubbe, M. A., \& Panczyk, M. (2007). Dewatering of refined, bleached hardwood kraftpulp by gravity, vacuum, and centrifugation with applied pressure part 2 . effects of wetend additives. [Desaguamento de polpa kraft branqueada de fibra curta refinada: Por gravidade, vácuo e centrifugação com aplicação de pressão Parte 2. Efeitos dos aditivos da parte úmida] O Papel (Brazil), 68(10), 88-100.

14) lakovlev, M., Hiltunen, E., \& van Heiningen, A. (2010). Paper technical potential of spruce SO2-ethanol-water (SEW) pulp compared to kraft pulp. Nordic Pulp and Paper Research Journal, 25(4)

15) lotti, M., Gregersen, $\varnothing$ W., Moe, S., \& Lenes, M. (2011). Rheological studies of microfibrillar cellulose water dispersions. Journal of Polymers and the Environment, 19(1), 137-145.

16) Isogai, A., Saito, T., \& Fukuzumi, H. (2011). TEMPO-oxidized cellulose nanofibers. $\mathrm{Na}$ - 
noscale, 3(1), 71-85.

17) K.Dimic-Misic, A.Puisto, M.Alava, J. Paltakari, T. Maloney. (2013). The influence of shear on the dewatering of high consistency nanofibrillated cellulose furnishesCellulose1-12 (2012),

18) K.Dimic-Misic, A.Puisto, P.Gane, K.Nieminen ,M.Alava, J. Paltakari, T. Maloney. (2013). The role of MFC/NFC swelling in the rheological behaviour and dewatering of high consistency furnishes.

19) Kang, T., \& Paulapuro, H. (2006). Effect of external fibrillation on the retention of filler.Kami $\mathrm{Pa}$ Gikyoshi/Japan Tappi Journal, 60(8), 88-92.

20) Karppinen, A., Vesterinen, A. H., Saarinen, T., Pietikäinen, P., \& Seppälä, J. (2011). Effect of cationic polymethacrylates on the rheology and flocculation of microfibrillated cellulose. Cellulose, 18, 1381-1390.

21) Lasseuguette, E., Roux, D., \& Nishiyama, Y. (2008). Rheological properties of microfibrillar suspension of TEMPO-oxidized pulp. Cellulose, 15(3), 425-433.

22) Lindstrom, T. (1985). CELLULOSE SUSPENSIONS AND POLYELECTROLYTES - SOME KINETIC ASPECTS. 121-122.

23) Marrucci, G. (1996). Dynamics of entanglements: A nonlinear model consistent with the cox-merz rule. Journal of Non-Newtonian Fluid Mechanics, 62(2), 279-289.

24) Mishra, S. P., Thirree, J., Manent, A., Chabot, B., \& Daneault, C. (2010). Ultrasound-catalyzed TEMPO-mediated oxidation of native cellulose for the production of nanocellulose: Effect of process variables. BioResources, 6(1), 121-143.

25) Nakagaito, A. N., Fujimura, A., Sakai, T., Hama, Y., \& Yano, H. (2009a). Production of microfibrillated cellulose (MFC)-reinforced polylactic acid (PLA) nanocomposites from sheets obtained by a papermaking-like process. Composites Science and Technology, 69(7), 1293-1297.

26) Nakagaito, A. N., Fujimura, A., Sakai, T., Hama, Y., \& Yano, H. (2009b). Production of microfibrillated cellulose (MFC)-reinforced polylactic acid (PLA) nanocomposites from sheets obtained by a papermaking-like process. Composites Science and Technology, 69(7-8), 1293-1297.

27) Ovarlez, G., Rodts, S., Chateau, X., \& Coussot, P. (2009). Phenomenology and physical origin of shear localization and shear banding in complex fluids. Rheologica Acta, 48(8), 831-844
28) Puisto, A., Illa, X., Mohtaschemi, M., \& Alava, M. (2012a). Modeling the viscosity and aggregation of suspensions of highly anisotropic nanoparticles. The European Physical Journal E: Soft Matter and Biological Physics, 35(1), 1-7

29) Puisto, A., Illa, X., Mohtaschemi, M., \& Alava, M. (2012b). Modeling the rheology of nanocellulose suspensions. Nordic Pulp and Paper Research Journal, 27(2), 277.

30) Richmond, F. (2012). The coating of nanofibrillated cellulose onto paper using flooded and metered size press methods. 12PaperCon/Papers/12PAP18.Aspx,

31) Richmond, Finley - Co, Abert - Bousfield Douglas. (2012). The coating of nanofibrillated cellulose onto paper using flooded and metered size press methods. 12PaperCon/ Papers/12PAP18.Aspx

32) Saito, T., \& Isogai, A. (2005). TEMPO-mediated oxidation of native cellulose. Appita Annual Conference, 3, 337-340

33) Siró, I., \& Plackett, D. (2010). Microfibrillated cellulose and new nanocomposite materials: A review. Cellulose, 17(3), 459-494.

34) Subramanian, R., Hiltunen, E., \& Gane, P. A. C. (2011). Potential use of micro-and nanofibrillated cellulose composites exemplified by paper. Cellulose Fibers: Bio-and Nano-Polymer Composites: Green Chemistry and Technology, 121

35) Turbak, A. F., Snyder, F. W., \& Sandberg, K. R. (1984). Microfibrillated Cellulose-A new composition of commercial significance. Atlanta, Ga., USA. 115-124.

36) Xiong, X., Guo, S., Xu, Z., Sheng, P., \& Tong, P. (2009). Development of an atomic-force-microscope-based hanging-fiber rheometer for interfacial microrheology. Physical Review E - Statistical, Nonlinear, and Soft Matter Physics, 80(6)

37) Yılmaz, O., Cheaburu, C. N., Gülümser, G., \& Vasile, C. (2011). Rheological behaviour of acrylate/montmorillonite nanocomposite latexes and their application in leather finishing as binders. Progress in Organic Coatings, 70(1), 52-58.

Paper sent to revision: 15.01.2013.

Paper ready for publication: 28.08.2013. 